\section{Case Reports in Oncology}

\title{
Pneumothorax during Pemetrexed Treatment in a Patient with Non-Small Cell Lung Cancer: A Case Report and Literature Review
}

\author{
Yoshiro Nakahara ${ }^{a, b}$ Shinichiro Mikura ${ }^{b}$ Makoto Nagamata ${ }^{b}$ \\ Tomoya Fukui $^{a}$ Jiichiro Sasaki ${ }^{c}$ Noriyuki Masuda ${ }^{a}$ \\ ${ }^{a}$ Department of Respiratory Medicine, Kitasato University School of Medicine, \\ Sagamihara, Japan; ${ }^{b}$ Department of Thoracic Oncology and Respiratory Medicine, Tokyo \\ Metropolitan Cancer and Infectious Diseases Center, Komagome Hospital, Tokyo, Japan; \\ ${ }^{c}$ Research and Development Center for New Medical Frontiers, Kitasato University School \\ of Medicine, Sagamihara, Japan
}

\section{Keywords}

Pemetrexed $\cdot$ Pneumothorax $\cdot$ Non-small cell lung cancer

\begin{abstract}
Pemetrexed is a multitargeted antifolate that has demonstrated antitumor activity in nonsmall cell lung cancer. A 70-year-old male presented with a stage IV non-small cell lung cancer. The patient was treated with pemetrexed as third-line chemotherapy. However, a pneumothorax occurred 16 days after the administration of the second cycle of pemetrexed. The pneumothorax was slight and the patient was observed without undergoing any additional treatment. Twenty-four days after its initial occurrence, the pneumothorax had improved. This is the first case of pneumothorax that has been observed during pemetrexed treatment. Pneumothorax during chemotherapy is rare; however, it is a life-threatening complication and should not be overlooked.

(C) 2017 The Author(s)

Published by S. Karger AG, Basel
\end{abstract}


 Oncology}

\section{Introduction}

Pemetrexed is a multitargeted antifolate that has demonstrated antitumor activity in non-small cell lung cancer. It has several notable adverse effects including myelosuppression, nausea, vomiting, diarrhea, and skin toxicity. However, the occurrence of pneumothorax during pemetrexed chemotherapy has not been previously reported [1]. Herein, we present a case of pneumothorax that was observed during pemetrexed chemotherapy.

\section{Case Report}

An asymptomatic 70-year-old man was referred to the Department of Respiratory Diseases because of abnormal shadows found during a chest computed tomography scan. The scan revealed bilateral infiltrative shadows in the lower lung lobes (Fig. 1). Transbronchial lung biopsy was performed and the patient was diagnosed with non-small cell lung cancer classified as stage IV (T3N3M1a). The patient was treated every 3 weeks with 3 cycles of first-line chemotherapy, including carboplatin (AUC 5) on day 1 and gemcitabine $(1,000$ $\mathrm{mg} / \mathrm{m}^{2}$ ) on days 1 and 8 . After 3 cycles of chemotherapy, the bilateral infiltrative shadows had progressed. Consequently, the patient was treated every 3 weeks with 3 cycles of docetaxel $\left(60 \mathrm{mg} / \mathrm{m}^{2}\right)$ monotherapy as second-line chemotherapy. However, after the third cycle, the infiltrative shadows had progressed again. Subsequently, the patient was treated with pemetrexed $\left(500 \mathrm{mg} / \mathrm{m}^{2}\right)$ as third-line chemotherapy, every 3 weeks. In the first cycle of the chemotherapy, there were no grade 3 or 4 adverse events. However, the patient experienced sudden onset of cough and dyspnea 16 days after the administration of the second cycle of pemetrexed. Physical examination showed decreased breathing sounds in the right chest, and a chest radiograph revealed a pneumothorax (Fig. 2, Fig. 3). Because the pneumothorax was slight, the patient was observed without any treatment, and pemetrexed therapy was stopped. The pneumothorax had improved 24 days after the occurrence of the pneumothorax (Fig. 4). However, the patient died 2 months after the occurrence of pneumothorax because of disease progression.

\section{Discussion}

Secondary spontaneous pneumothorax after chemotherapy is rare; however, welldocumented occurrence is associated with primary or metastatic lung lesions. Lai et al. [2] reported that pneumothorax appeared in 18 out of 5,567 (0.32\%) lung cancer patients, and 2 out of 18 patients experienced pneumothorax during chemotherapy. Maniwa et al. [3] reported that pneumothorax associated with the treatment for pulmonary malignancy (primary and metastatic) tends to require chest tube drainage over a long period, and sometimes requires surgical treatment; this is associated with increased perioperative morbidity and mortality because of the dense adhesions or anatomical changes in the thoracic cavity caused by treatment or the progression of the tumors [3].

Recently, molecular targeted therapies have also been linked with the occurrence of pneumothorax. Mori et al. [4] reported a case of simultaneous bilateral spontaneous pneumothorax that occurred during gefitinib treatment for lung adenocarcinoma with multiple lung metastases. A case of pneumothorax after bevacizumab-containing chemotherapy for colorectal cancer with lung metastases was reported by Yang et al. [5]. In addition, Gennatas 
et al. [6] reported a case of pneumothorax during crizotinib therapy in a patient with anaplastic lymphoma kinase-rearranged lung adenocarcinoma. In contrast, pneumothorax during cytotoxic chemotherapy treatment is rare, and secondary spontaneous pneumothorax during pemetrexed has not been previously reported in the available literature.

The mechanism of secondary pneumothorax from pulmonary malignancy is unclear, although several theories have been proposed. The proposed mechanisms are as follows: accidental rupture of the subpleural bulla or bleb during chemotherapy; the formation of a bronchopleural fistula as a result of direct tumor invasion or necrosis of the peripheral tumor after effective chemotherapy; a check-valve mechanism associated with airway compression by the tumor; and an air leak caused by pulmonary infarction from tumor emboli [7].

In the present case, there was no clinical response to pemetrexed treatment, and tumor necrosis was not observed. Furthermore, neither progression of airway compression or signs of pulmonary infarction were observed. In addition, there were no subpleural bullae or blebs on chest computed tomography before pemetrexed treatment. We believe that the pneumothorax was possibly caused by the formation of a bronchopleural fistula as a result of direct tumor invasion of the peripheral tumor.

In summary, ours is the first reported case of pneumothorax to have occurred during pemetrexed treatment. Pneumothorax during chemotherapy is rare; however, it is a lifethreatening complication that should not be overlooked. It is important to observe the patients' symptoms during chemotherapy treatment.

\section{Statement of Ethics}

The authors have no ethical conflicts to disclose.

\section{Disclosure Statement}

The authors state that they have no conflict of interest.

\section{References}

1 Hanna N, Shepherd FA, Fossella FV, et al: Randomized phase III trial of pemetrexed versus docetaxel in patients with non-small-cell lung cancer previously treated with chemotherapy. J Clin Oncol 2004;22:1589-1597.

2 Lai RS, Perng RP, Chang SC: Primary lung cancer complicated with pneumothorax. Jpn J Clin Oncol 1992;22:194-197.

-3 Maniwa T, Nakagawa K, Isaka M, et al: Pneumothorax associated with treatment for pulmonary malignancy. Interact Cardiovasc Thorac Surg 2011;13:257-261.

4 Mori M, Nakagawa M, Fujikawa T, et al: Simultaneous bilateral spontaneous pneumothorax observed during the administration of gefitinib for lung adenocarcinoma with multiple lung metastases. Intern Med 2005;44:862-864.

5 Yang SH, Lin JK, Chen WS, et al: Pneumothorax after bevacizumab-containing chemotherapy: a case report. Jpn J Clin Oncol 2011;41:269-271.

6 Gennatas S, Stanway SJ, Thomas R, et al: Early pneumothorax as a feature of response to crizotinib therapy in a patient with ALK rearranged lung adenocarcinoma. BMC Cancer 2013;13:207.

7 Srinivas S, Varadhachary G: Spontaneous pneumothorax in malignancy: a case report and review of the literature. Ann Oncol 2000;11:887-889. 


\section{Case Reports in Oncology}

www.karger.com/cro

Nakahara et al.: Pneumothorax during Pemetrexed Treatment in a Patient with NonSmall Cell Lung Cancer: A Case Report and Literature Review

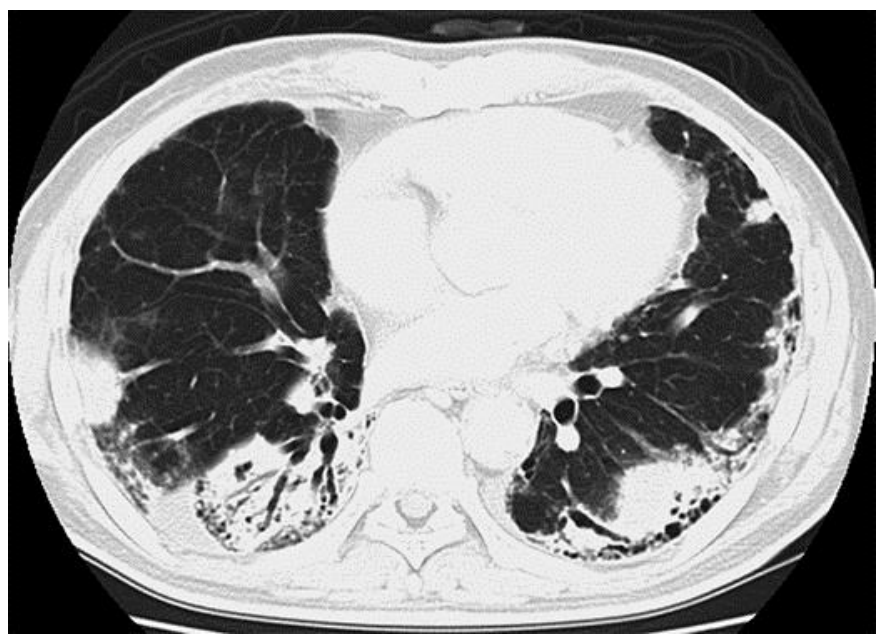

Fig. 1. Chest computed tomography demonstrating bilateral infiltrative shadows. These lesions were diagnosed as non-small cell lung cancer by transbronchial lung biopsy.

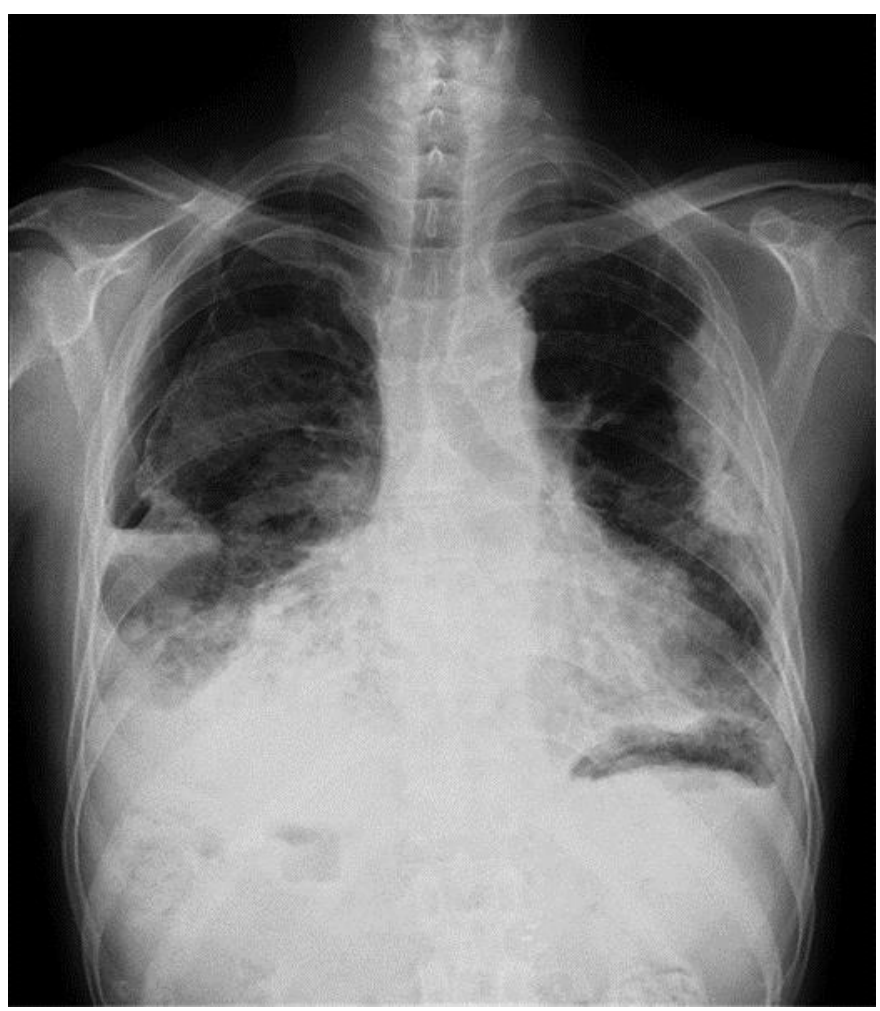

Fig. 2. Chest X-ray demonstrating right pneumothorax. 


\section{Case Reports in Oncology}

Nakahara et al.: Pneumothorax during Pemetrexed Treatment in a Patient with NonSmall Cell Lung Cancer: A Case Report and Literature Review

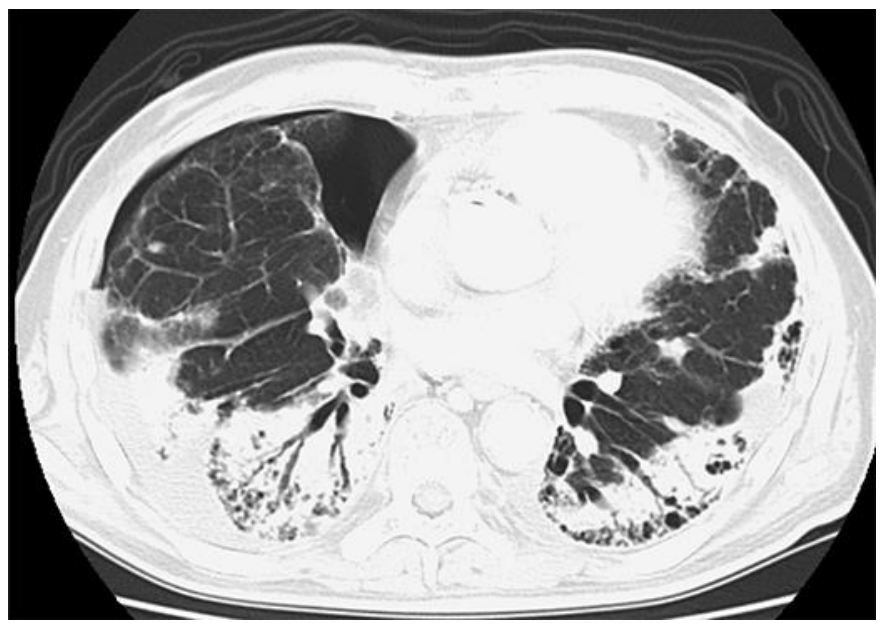

Fig. 3. Chest computed tomography demonstrating right pneumothorax, and bilateral infiltrative shadows had progressed.

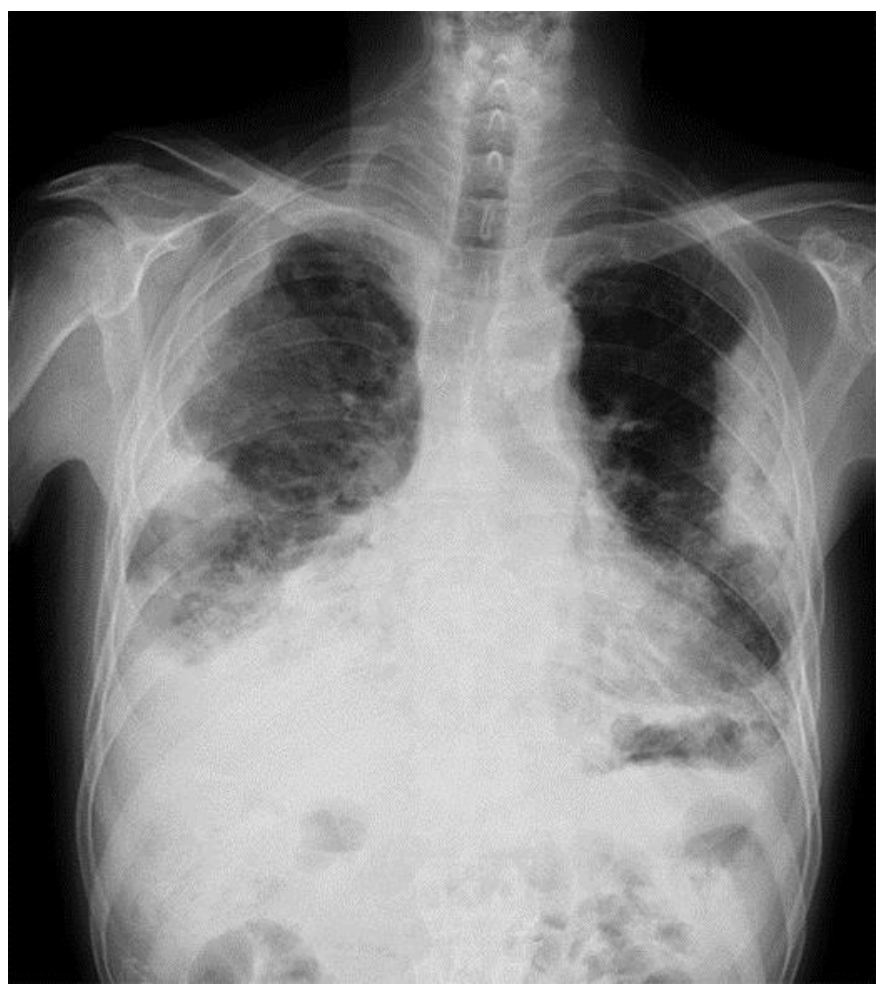

Fig. 4. The pneumothorax had improved 24 days after the occurrence of the pneumothorax. 